\title{
Solar Navigational Planning for Robotic Explorers
}

\author{
Kimberly Shillcutt* \\ NASA Johnson Space Center \\ 2101 NASA Rd. 1, Code ER2 \\ Houston, TX 77058 \\ kimberly.j.shillcutt1@jsc.nasa.gov
}

William Whittaker

Field Robotics Center, The Robotics Institute

Carnegie Mellon University

Pittsburgh, PA 15213

red@ri.cmu.edu

navigational tasks such as coverage patterns and longrange path plans. By evaluating tasks with respect to sun, terrain and time considerations, exploration robots can select plans with a better chance of survival and success.
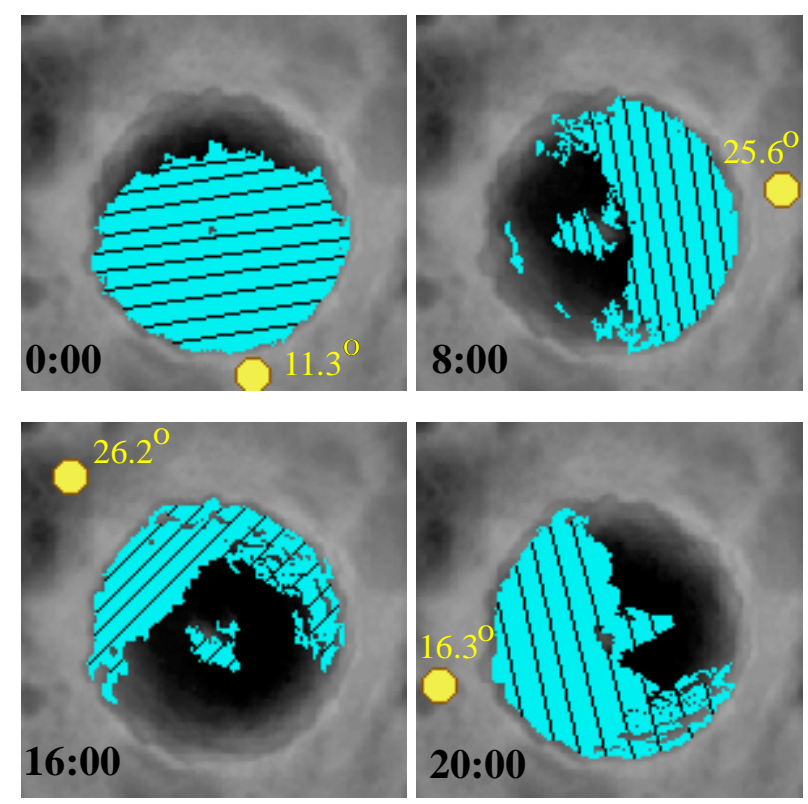

Figure 1: Motion of shadows at $80 \mathrm{~S}$, Earth's summer

Robotic explorers are well-suited to take advantage of a changing environment, as they have the ability to travel to seek out the most beneficial conditions. Onboard knowledge of terrain, lighting, and Earth visibility are essential for finding locations and orientations for recharging batteries or communicating with Earth. With sufficient knowledge of the terrain and the sun, robots can continually seek sunlit valleys and ridges, generating power to accomplish goals and remain alive as long as possible. This ability is particularly notable in polar regions, where the sun is continually above the horizon for long periods of time, and can be only barely below the horizon at other times [2]. Here, robots can follow the sun around planetary poles or terrain features, continually staying in sunlight. By finding and following such routes, a robotic explorer can operate in polar regions for an entire season or more, remaining active continually without the need to pause at nightfall and shut down non-critical systems, greatly extending the typical lifetime of planetary robots.

*This research performed while author was at Carnegie Mellon University. 


\section{Approach}

\subsection{Task Simulation}

To address the question of how robotic explorers can benefit from sun, terrain and time information, a set of task simulations was developed. Environmental and robotic modeling enables potential navigational tasks to be simultaneously evaluated in a range of categories as the simulations are performed. Considering the attributes most needed for a particular mission, the best navigational task can be selected based on the resulting evaluations for a given set of tasks.

Exploration requires driving throughout a region, and a systematic method is generally most efficient for covering an area. Coverage patterns are one primary task type developed for this research. The most common pattern is driving back and forth in parallel straight rows. A variety of other patterns have also been developed for this work, each with specific characteristics that may prove more or less desirable given the mission and the environmental situation. Spiral patterns are another obvious type, while curved patterns are similar to the straight rows pattern but with a range of possible row curvatures. Sun-following patterns are designed specifically for polar regions, with low sun angles and near-vertical solar panels on either side of a robot. In this pattern, a robot continually turns so that one solar panel is always facing the sun. Simulated ground tracks of the patterns are shown in Figure 2.



Figure 2: Coverage pattern simulated groundtracks

By simulating each type of pattern under the relevant sun, terrain and time conditions, the best pattern for the conditions can be found. The patterns are defined incrementally, simulating a robot moving along the pattern one (simulated) second at a time. A structural loop is initiated in which the pattern-following function is called once during every pass through the loop, until the loop is terminated by the completion of the task.

\subsection{Task Evaluation}

Evaluation functions are called once during every pass through the task simulation loop, keeping track of the desired costs and benefits throughout the performance of the task [5]. For instance, the evaluation function for the total time required to perform a task is a single step: incrementing the time by one second. Other evaluation algorithms are more complicated, and include functions for solar energy generation, energy consumption, area covered, and overlap of covered area.

Once all desired evaluation totals are calculated for a pre-determined set of potential tasks, they can be compared to select the best task. If one evaluation category is of prime importance, such as power generation, then the task generating the most power can be selected, or else a weighted sum of multiple evaluations can be used instead. Alternately, tasks can be filtered through cutoff value tests for one or more categories, with surviving tasks ranked by the remaining categories.

Values needed to calculate solar energy generation are the incident angle of the sun on a solar panel, and the amount of power which can be generated by that panel. Environmental modeling consists of calculating the orbital parameters of the Earth-sun-moon system, and incorporating terrain features to determine shadowing. A solar ephemeris algorithm was developed, producing accurate values for the sun's altitude and azimuth, and optionally the Earth's, with respect to a robot's current location on the Earth or moon. This calculation involves detailed geometrical transformations described in [6], using information in [4] combined with readily available algorithms for basic sun and moon positions. By comparing terrain elevations in the direction of the sun, any occlusions of sunlight can be determined (see Figure 3). Terrain information is obtained from digital elevation maps, which are read into an internal grid-based map when first initialized. Details, including finding great circles, incorporating surface curvature, and geometrically extending past the edge of a known map, are given in [6].

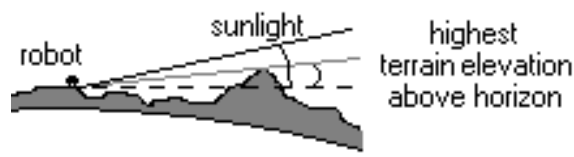

\section{Figure 3: Comparing terrain elevations to sun}

Robot configuration modeling requires knowing the geometrical normals to each solar panel relative to the robot. These normals are rotated based on the robot's current pose, producing the normals in world coordinates relative to the sun. The solar power varies directly with 
the cosine of the angle between each panel's normal and the direction of the sun. Many of the simulations in this work are based on the two fixed solar panels attached to the sides of the robot Nomad used in field work (see Figure 4). Details such as reflection and atmospheric diffusion of light are not included in this simulation, but can be calibrated for, as described in section 3.1.

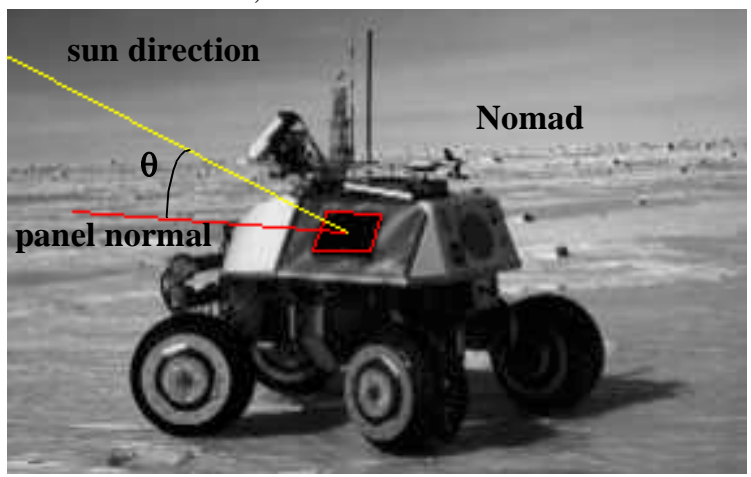

Figure 4: Nomad solar panel normal, sun angle

One problem which may occur is that the visibility calculations assume the ground, and therefore the robot, is flat and tangent to the planetary sphere. Terrain slopes will violate this assumption and invalidate the angular calculations. At runtime this is not a problem as the robot's sensors give the correct pose to incorporate into the calculations. For predictive simulations, several solutions are possible. One, a digital elevation map or other remote sensing data can be processed to estimate average terrain slopes, though this may prove inaccurate with lowresolution maps. Secondly, for regions in which the slope is roughly constant, such as on the side of a hill, and for which the entire pattern will be carried out in that region, the robot can determine the slope ahead of time with sensors. This is one benefit of having a solar ephemeris library on board the robot, as real-time data can be immediately incorporated. However, when only the existence of sunlight is sought, such as for sunlit path searches, knowledge of terrain slopes is not required.

The evaluation function for energy consumption is based on statistical telemetry from field tests with the robot Nomad. Power consumption values were filtered and split into categories, with significant trends found for point turns, changing turning radii, and high or low pitch. Based on these trends, the evaluation takes the current pose, commanded turning radius, and previously commanded turning radius, calculating energy consumption for each pass through the simulation loop.

The amount of area covered or overlapped by a robot depends on the "footprint" of the sensor being used, typically a camera. The evaluation takes the current pose and calculates which pixels in the internal grid-based map are seen by a camera with a given field of view. To calculate the incremental area covered, the algorithm sums the areas of all currently visible pixels which have not previously been marked. To calculate the overlap, or amount of area seen more than once, the algorithm sums the areas of currently visible pixels which have already been marked. If a pixel has only recently been marked, it is not added in order to avoid the problem of double counting before a robot has moved far enough away.

These evaluation models may be inadequate for some situations, but the simulation and evaluation structure can easily incorporate any new, more accurate models, such as energy consumption models including terrainability or wind resistance. The task algorithm loop is also used for actual robot motion, during which both telemetry and evaluation function results are recorded once a second. As opposed to a priori simulations, where evaluation results are generated in a fraction of the actual task time, concurrent simulations evaluate the patterns as they are being performed. These evaluations are compared to actual telemetry, to aid in calibration of models, generation of statistical data, and comparison with ground truth.

\subsection{Solar Navigation}

A second type of task studied in this work is solar navigation: sun-synchronous and sun-seeking paths. Sunsynchronous paths enable robots to continually generate enough power to remain active, while sun-seeking paths enable robots working in shadow to find the closest areas which will be sunlit (or Earthlit) for a specified length of time. Both types of paths can be found using the solar ephemeris library developed for this research, either onboard during missions, or off-board for more time and memory intensive calculations.

One useful calculation is finding and displaying shadows for an entire region and range of times. A sequence of these shadow snapshots combined into an animation graphically shows shadow movements across the region. Pre-computing shadow maps and combining them into a database also enables efficient path searches, combining two-dimensional aspects of a region with the third temporal dimension. A sunlight (or Earthlight) endurance map augments two dimensional terrain map pixels with a linked list of time periods, with each period containing fields for starting and stopping times and the state of sunlight or shadow. In most cases, these periods will be few in number and fairly continuous, due to the regularity of the sun's motion.

Sun-synchronous path finding involves an 8connected wavefront propagation search through twodimensional space, starting from a specific pixel and time. Search time is reduced by considering only pixels in the direction of desired travel, and within a given range of a desired center line of travel. The existence of shadowing in a pixel is determined for the time at which the robot could reach that pixel, given an expected speed. Shadow 
determination can be made by running the solar ephemeris and terrain algorithm for each pixel, or by accessing the appropriate portion of a pre-computed database.

Sun-seeking path finding also involves a wavefront propagation search, using a sunlight endurance map to check the status of each pixel. The time at which a robot will arrive at a pixel can be compared to the pixel's linked list to determine in which time period the arrival time falls, and whether that time period is sunlit or shadowed. If sunlit, the period stopping time can be consulted to determine the time left in that location before the sun is occluded. If the remaining sunlight time is not sufficient for the robot to recharge, the search continues. Similarly, locations with Earth visibility can be sought to reinstate communications with users. More implementation details and restrictions can be found in [6].

\section{Field Results}

\subsection{Robotic Antarctic Meteorite Search}

Much of this research was implemented on the robot Nomad for the Robotic Antarctic Meteorite Search. This project involved performing coverage patterns on ice fields, searching for meteorites with a variety of sensors. Solar panels were attached to the robot for testing purposes only. Telemetry data provided verification of pattern following and solar power generation, from tests in Pittsburgh, Williams Field near McMurdo, Antarctica, and at a remote site called Elephant Moraine 160 miles from McMurdo. Further results are described in [1]. Path planning incorporating shadows was tested only in simulation, as the Antarctic field test locations were primarily flat, with very little shadowing.

\subsection{Solar Power Generation}

The solar based selection of plans depends heavily on the accuracy of solar power generation predictions. This section shows that the predictions are accurate once the solar panel models are properly calibrated, with a few initial measurements to adjust and scale the simulation. The actual power produced is compared with predicted values for each panel, for the two cases of facing toward and away from the sun. This produces a scaling factor and minimum value to be applied to predictions, assuming the atmospheric conditions remain similar. Figure 5 shows the results of applying the calibration to concurrently simulated power values for two coverage patterns, compared to actual values, both for a sunny and a cloudy day. The square wave effect is due to the alternating row directions of the straight rows pattern.

Calibrated a priori simulations are shown in Figure 6 along with actual results from Elephant Moraine and Williams Field, with plots of cumulative solar energy generated from both panels. The total simulation times differ from the actual patterns due to variable robot speed, particularly during turns. Note that the two coverage pattern types are clearly distinguishable from one another. The mean error of the simulations as a percent of final energy values for Elephant Moraine is $0.65 \%$ for the straight rows and $1.25 \%$ for the spiral, while for Williams Field, the errors are $0.19 \%$ and $1.62 \%$.

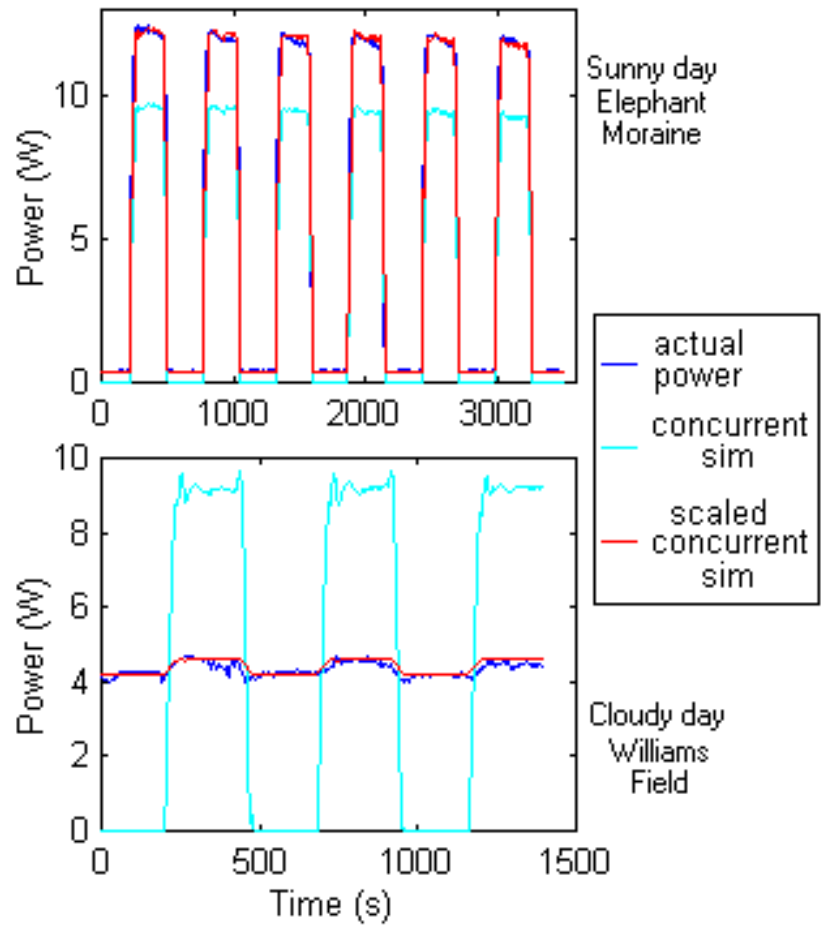

Figure 5: Calibrated solar power values
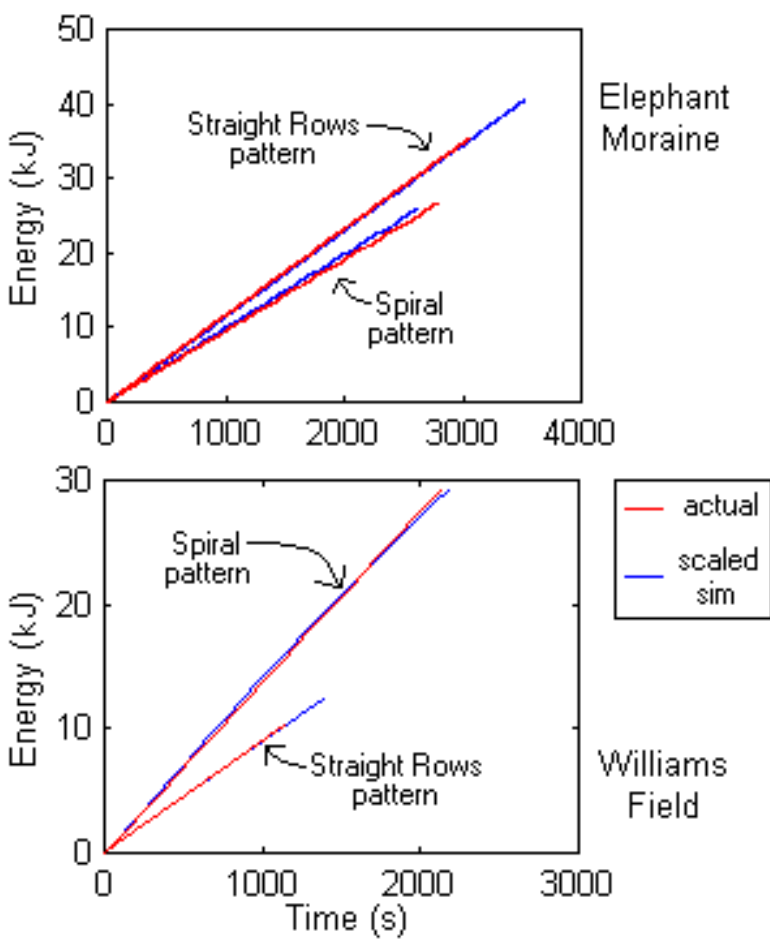

Figure 6: Calibrated sims for multiple patterns 


\section{Simulation Results}

A wide variety of patterns were simulated to gain an understanding of their characteristics, and to develop heuristics for selecting subsets of plan types, shortening evaluation times by reducing the needed number of patterns, starting times and locations to evaluate on-board. Without shadowing, energy generation results of straight rows patterns varied sinusoidally with the initial robot heading. Most evaluation results of curved patterns varied directly with row curvature, allowing analytical equations to generate evaluations without simulation.

When shadowing was added, similarities in solar energy generation totals were found for straight rows patterns starting on the same side of an area, and moving along the same direction, for a given time. For instance, north-south patterns generated approximately the same energy whether the pattern started in the northwest or southwest corners, while a different total was produced by patterns starting in the northeast or southeast corners. This is because the time spent traveling along a single row is usually negligible with respect to the motion of the sun, while the progression across the width of a large area is more affected by the sun's motion. In these simulations, the difference between opposite starting corners resulted in an energy difference of up to $39 \%$.

Pose uncertainty was also simulated to determine the magnitude of the effect on power evaluations. Randomized roll and pitch values of up to 8 degrees were simulated, with minor resulting effects on solar power generation. Differences between different pattern types were larger than the differences with and without pose variations. More details on these and other simulations are described in [6].

\subsection{Mission Scenarios}

More involved mission scenarios were developed, assuming a robot derived power from solar panels and a battery, and required recharging stops whenever its battery reached a critical level. The model assumed the robot either generated more power than it consumed, or drained power from the battery.

Three missions scenarios were simulated. In one, the robot drove for a certain total distance. In the other two, the robot drove that distance with random targets included once every 100 meters or so, stopping to "investigate" for about 5 minutes, either halting in place or else turning to an optimal power generating orientation. 23 patterns of all types were tried, for a range of latitudes and locations. Total mission times required, the amount of time before the first recharging, the time spent recharging, and the number of recharging periods required were recorded.

To gain an understanding of numerical improvements possible by choosing one pattern type over another, three metrics were considered. Lifetime is the length of time before recharging is first needed. Productivity is the percent of time spent driving or looking at targets out of the total mission time. Efficiency is the percent of total mission time saved compared to the worst case pattern. From the simulations performed for Earth and moon locations, robot lifetimes improved by up to $161 \%$, robot productivity improved by over $47 \%$, and robot efficiency improved by over $58 \%$.

\subsection{Solar Navigation}

Sun-synchronous paths were found around a terrain feature of about $7 \mathrm{~km}$ circumference in the Canadian Arctic, for a robot traveling $10 \mathrm{~cm} / \mathrm{s}$. Autonomous identification of these paths was done off-board with a search algorithm considering both the passage of shadows over time and the continual progression of the robot. The path shown in Figure 7 was constrained to be between 940 and 1440 meters from the center, and to travel clockwise, following the sun. The path ended upon reaching the same angular position as the start. The sun's initial position is shown at the starting time of $8 \mathrm{pm}$ local time. The waypoints marked on the image indicate equally spaced one-hour time increments, with the exception of the first interval, which is a half hour.

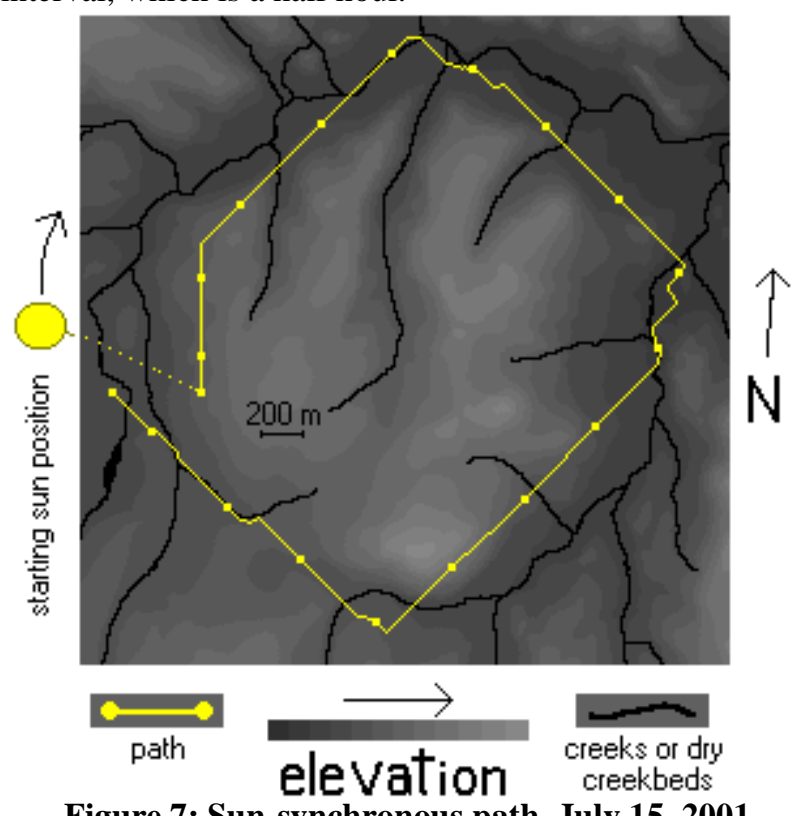

Figure 7: Sun-synchronous path, July 15, 2001

The preponderance of diagonal path lines is due to the search being 8-connected, and being breadth-first. The first possible path, with the fewest number of steps, is found, and a diagonal step is the shortest way to get from one pixel to another. Situations where shadows have to be avoided are obvious, with deviations from the diagonals. The current implementation does not take into account terrain navigability, such as steepness of slopes, and ignores the creeks, treating them as safe ground. 


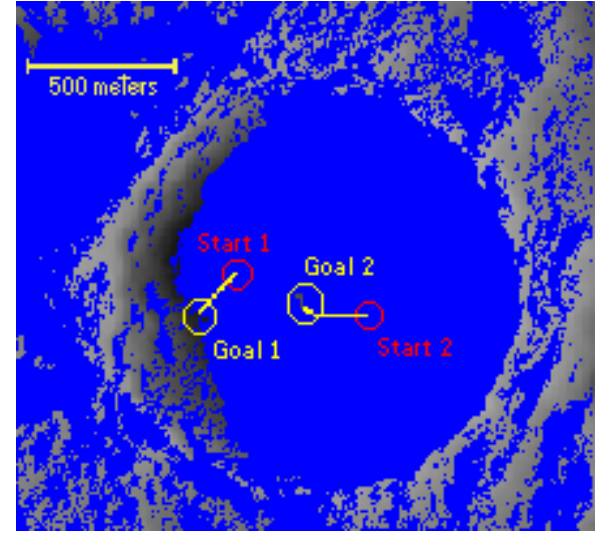

Figure 8: Sun-seeking paths, Jan 15, 2000

Sun-seeking navigation is shown in Figure 8, where two autonomously-generated paths are shown taking a robot from shadowed crater locations to sunlit points near the crater wall or central peak. The goal points will remain sunlit long enough to recharge the robot's batteries. The shadow snapshot image shows the position of shadows, colored blue, at the starting time of the path. The crater is actually the moon's Tycho crater "placed" on Earth, centered at $80 \mathrm{~S}$ latitude [3]. The range of elevations is from -40 meters to nearly 500 meters high. The paths will take about half an hour for a robot traveling $15 \mathrm{~cm} / \mathrm{s}$.

\section{Conclusions}

This research shows that solar information can be highly profitable for robotic explorers, with multiple ways to utilize such information. On-board sun, terrain and time information enables intelligent planning by the robot, while off-board information enables long-range planning and the generation of databases for use by either robotic explorers or humans.

\subsection{Significance}

Several key points contribute to the advancement of robotic exploration and navigational planning:

New robotic navigational abilities. The ephemeris algorithm enables new robotic capabilities of following sun-synchronous and sun-seeking paths, empowering uninterrupted robot activity, extending lifetimes, and enabling autonomous recovery from power and communication losses.

On-board robotic planning structure incorporating environmental, time-dependent modeling. This structure models and evaluates sun and terrain effects during navigational tasks, most notably power generation, and can easily be expanded to include additional or more precise environmental and robot configuration models. Temporal aspects are integrated through step-by-step simulation of tasks.

Solar ephemeris algorithm applied to terrain elevation maps and robotic planners. This algorithm accurately determines orbital parameters and shadowing for any given Earth or lunar location, date and time, and can be used as an analytical planning tool in real-time predictive task evaluations.

Detailed analysis of coverage patterns. Basic characteristics of new coverage patterns are analyzed, reducing the set of potential patterns for evaluation.

\subsection{Future Work}

More detailed robot and environment models can be incorporated into the structure developed in this research. Power consumption including measures of terrainability is one desirable area. Wind power generation has also been studied for this work, but lacks consideration of wind resistance and its effect on power consumption.

Least-cost searches such as $A^{*}$ can be used for sunsynchronous and sun-seeking path searches, adding costs of terrainability and turning as well as more accurate path distances. An alternative approach is to use pre-computed, discretized databases of shadow maps as a threedimensional search space. The result can be visualized as a hopefully connected region of sunlit areas, with blobs of shadows interspersed. This space can be approached with a number of search algorithms to produce viable paths. Searches must be restricted to only allow one-way travel in the temporal dimension, but can be more flexible in allowing variable robot speeds throughout the path.

\section{Acknowledgements}

The members of the Robotic Antarctic Meteorite Search project, in the Field Robotics Center at Carnegie Mellon University, contributed immeasurably to this research. Partial support was also provided by a NASA Graduate Student Researcher fellowship, NGT-5-50248.

\section{References}

1 Apostolopoulos, D., et al., "Technology and Field Demonstration of Robotic Search for Antarctic Meteorites," International Journal of Robotics Research, December 2000.

2 Bussey, D., P. Spudis, M. Robinson, "Illumination conditions at the lunar south pole," Geophysical Research Letters, Vol. 26, No. 9, May 1999.

3 Margot, J. L., et al., "The Topography of Tycho Crater", Journal of Geophysical Research, Vol. 104, No. E5, May 1999.

4 Meeus, Jean, Astronomical Algorithms, WillmannBell: Richmond, VA, 1991.

5 Shillcutt, Kimberly and William Whittaker, "Modular Optimization for Robotic Explorers," Integrated Planning for Autonomous Agent Architectures, AAAI Fall Symposium, Orlando, FL, October 23-25, 1998.

6 Shillcutt, Kimberly, "Solar Based Navigational Planning for Robotic Explorers," Ph.D. Thesis, Robotics Institute, Carnegie Mellon Univ., Oct 2000. 